\title{
Adolf Karger
}

Equiaffine Darboux motions with double roots

Czechoslovak Mathematical Journal, Vol. 31 (1981), No. 1, 103-113

Persistent URL: http://dml.cz/dmlcz/101729

\section{Terms of use:}

(C) Institute of Mathematics AS CR, 1981

Institute of Mathematics of the Czech Academy of Sciences provides access to digitized documents strictly for personal use. Each copy of any part of this document must contain these Terms of use.

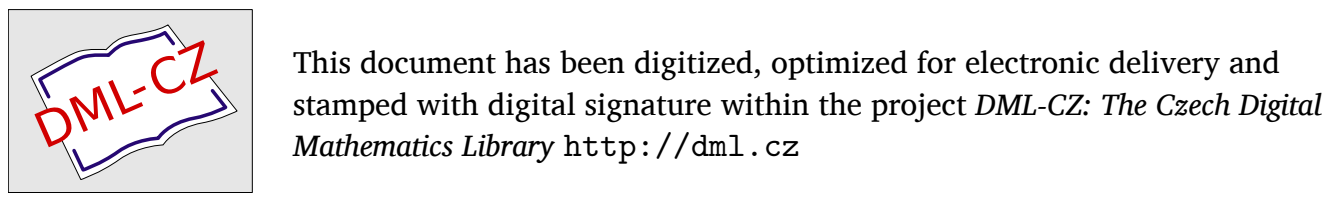




\title{
EQUIAFFINE DARBOUX MOTIONS WITH DOUBLE ROOTS
}

\author{
Adolf Karger, Praha
}

(Received September 19, 1979)

In the present paper we consider equiaffine space motions of one type only. The instantaneous motion of such a motion preserves a point $P$, a plane $\alpha$ and at least two straight lines $a$ and $b$, where $P=a \cap b, a \subset \alpha, b \neq \alpha$. We find all equiaffine space motions of this type which have only plane trajectories and all these trajectories are affinely equivalent (as parametrized curves). We give also explicit formulas for such motions.

Let us be given two equiaffine spaces $\boldsymbol{A}$ and $\bar{A}$ of dimension 3 with fixed frames $\mathscr{R}_{0}=\left\{0, f_{1}, f_{2}, f_{3}\right\}$ and $\overline{\mathscr{R}}_{0}=\left\{\overline{0}, \bar{f}_{1}, \bar{f}_{2}, \bar{f}_{3}\right\}$, respectively. Let $G$ be the group of all equiaffine transformations of $\boldsymbol{A}$ or $\overline{\boldsymbol{A}}$, regarded as the Lie group of all matrices of the form $g=\left(\begin{array}{ll}1, & 0 \\ T, & \gamma\end{array}\right)$, where $\gamma \in \operatorname{Sl}(3, \mathrm{R})$ and $T$ is a column with 3 entries.

The group $G$ acts also as the group of equiaffine transformations from $\bar{A}$ to $\boldsymbol{A}$ by the rule $g\left(\overline{\mathscr{R}}_{0}\right)=\mathscr{R}_{0} \cdot g$. In what follows, a frame will mean an equiaffine frame in $\boldsymbol{A}$ or $\overline{\boldsymbol{A}}$. An equiaffine motion is a curve in $G$, regarded as a one-parametric system of equiaffine transformations from the moving space $\bar{A}$ into the fixed space $\boldsymbol{A}$. In what follows we shall always suppose a sufficient degree of differentiability. By a lift of an equiaffine motion $g(t)$ we mean a set of pairs $(\mathscr{R}(t), \overline{\mathscr{R}}(t))$ of frames, $\mathscr{R}$ in $\boldsymbol{A}, \overline{\mathscr{R}}$ in $\bar{A}$, such that $g(t)(\overline{\mathscr{R}}(t))=\mathscr{R}(t)$. Let further $(\mathscr{R}(t), \overline{\mathscr{R}}(t))$ be any lift of a given equiaffine motion $g(t)$.

Denote $\mathscr{R}^{\prime}=\mathscr{R} \varphi, \overline{\mathscr{R}}^{\prime}=\overline{\mathscr{R}} \psi, 2 \omega=\varphi-\psi, 2 \eta=\varphi+\psi$. If $\bar{A}$ is a fixed point in $\bar{A}$, then $\bar{A}=\overline{\mathscr{R}} X$, where $X$ is the column of coordinates of $\bar{A}$ in $\bar{R}$. For the trajectory $g(\bar{A})$ of $\bar{A}$ in $\boldsymbol{A}$ we have $g(\bar{A})=\mathscr{R} X$. Denote by $\Omega_{k}$ the operator of the $k$-th derivative of the trajectory of a point $\bar{A}$ expressed in the frame $\mathscr{R}$ and write $g(\bar{A})^{(k)}=$ $=2 \mathscr{R} \Omega_{k} X$. By differentiation we get the following formulas:

$$
\Omega_{1}=\omega, \quad \Omega_{k+1}=(\omega+\eta) \Omega_{k}+\Omega_{k}(\omega-\eta)+\Omega_{k}^{\prime} .
$$

An equiaffine motion is called a Darboux motion iff

a) the trajectories of all points are plane curves and not all of them are straight lines,

b) all trajectories are affinely equivalent in the kinematical sense (with respect to the parameter $t$, see [1]). 
An equiaffine motion is a Darboux motion iff there are functions $\alpha_{1}(t)$ and $\alpha_{2}(t)$ such that (see [1])

$$
\Omega_{3}=\alpha_{1} \Omega_{1}+\alpha_{2} \Omega_{2} .
$$

Denote further

$$
\omega=\left(\begin{array}{ll}
0, & 0 \\
\omega_{0}, & \omega_{1}
\end{array}\right), \quad \eta=\left(\begin{array}{ll}
0, & 0 \\
\eta_{0}, & \eta_{1}
\end{array}\right), \quad \Omega_{k}=\left(\begin{array}{ll}
0, & 0 \\
\vartheta_{k}, & \Theta_{k}
\end{array}\right),
$$

where $\omega_{1}, \eta_{1}, \Theta_{k}$ are $3 \times 3$ matrices, $\omega_{0}, \eta_{0}, \vartheta_{k}$ are 3 -columns. We have always $\operatorname{Tr} \omega_{1}=\operatorname{Tr} \omega_{2}=0$ because $G$ preserves volumes. We shall also write $\omega=\left(\omega_{i j}\right)$, $\eta=\left(\eta_{i j}\right)$, where $i, j=0,1,2,3$ and of course $\omega_{0 i}=\eta_{0 i}=0$ for $i=0, \ldots, 3$. Substitution in (1) gives

$$
\begin{gathered}
\vartheta_{k+1}=\left(\omega_{1}+\eta_{1}\right) \vartheta_{k}+\Theta_{k}\left(\omega_{0}-\eta_{0}\right)+\vartheta_{k}^{\prime}, \\
\Theta_{k+1}=\left(\omega_{1}+\eta_{1}\right) \Theta_{k}+\Theta_{k}\left(\omega_{1}-\eta_{1}\right)+\Theta_{k}^{\prime} .
\end{gathered}
$$

If the lift of the motion is changed, we get new forms $\tilde{\omega}$ and $\tilde{\eta}$ instead of $\omega$ and $\eta$. The connection between new and old forms is

$$
\tilde{\omega}=h^{-1} \omega h, \quad \tilde{\eta}=h^{-1} \eta h+h^{-1} h^{\prime},
$$

where $h \in G$ is the change of the lift. Denote

Then

$$
h=\left(\begin{array}{ll}
1, & 0 \\
T, & \gamma
\end{array}\right)
$$

$$
\tilde{\omega}_{0}=\gamma^{-1}\left(\omega_{0}+\omega_{1} T\right), \quad \tilde{\omega}_{1}=\gamma^{-1} \omega_{1} \gamma .
$$

Formula (5) says that $\omega_{1}$ can be changed to a certain canonical form (if the characteristic roots of $\omega_{1}$ are real, we usually take the Jordan normal form as canonical). In the following we shall classify all Darboux motions for which $\omega_{1}$ has a double characteristic root. This means that after a suitable change of the parameter $t \omega_{1}$ will be one of the following matrices

$$
\text { i) } \omega_{1}=\left(\begin{array}{rrr}
1, & 1, & 0 \\
0, & 1, & 0 \\
0, & 0, & -2
\end{array}\right) \text {, ii) } \omega_{1}=\left(\begin{array}{rrr}
1, & 0, & 0 \\
0, & 1, & 0 \\
0, & 0, & -2
\end{array}\right) \text {. }
$$

(We notice that 0 cannot be a double characteristic root of $\omega_{1}$.) We see that in both cases i) and ii) $\omega_{1}$ is a regular matrix and so we can choose $T$ in (5) in such a way that $\tilde{\omega}_{0}=0$. This means geometrically that the motion has poloids. The instantaneous motion was described above.

In the following we shall consider only lifts of $g(t)$, for which $\omega_{1}$ is in the canonical form i) or ii) and $\omega_{0}=0$. Let us start with the case i): Denote by $H_{1}$ the isotropy group of lifts having the above mentioned properties. For $h \in H_{1}$ we then have 


$$
h=\left(\begin{array}{ll}
1, & 0 \\
T, & \gamma
\end{array}\right) \text { with } T=0, \quad \gamma=\left(\begin{array}{lll}
\alpha, & \beta, & 0 \\
0, & \alpha, & 0 \\
0, & 0, & \alpha^{-2}
\end{array}\right), \quad \alpha, \beta \in \mathrm{R}, \quad \alpha \neq 0
$$

Now we can change the lift of the motion using the elements from $H_{1}$ only. $\omega$ remains unchanged and $\eta$ will change according to (4) in the following way:

$$
\tilde{\eta}_{0}=\gamma^{-1} \eta_{0}, \quad \tilde{\eta}_{1}=\gamma^{-1} \eta_{1} \gamma+\gamma^{-1} \gamma^{\prime} .
$$

Substitution in the second formula of $(7)$ gives

$$
\tilde{\eta}_{1}=\left(\begin{array}{lll}
\eta_{11}-\alpha^{-1} \beta \eta_{21}, & \times, & \alpha^{-3}\left(\eta_{13}-\beta \alpha^{-1} \eta_{23}\right) \\
\eta_{21}, & \alpha^{-1} \beta \eta_{21}+\eta_{22}, & \alpha^{-3} \eta_{23} \\
\alpha^{3} \eta_{31}, & \alpha^{2}\left(\beta \eta_{31}+\alpha \eta_{32}\right), & \eta_{33}
\end{array}\right)+\gamma^{-1} \gamma^{\prime} .
$$

Substitution in (3) gives

$$
\begin{aligned}
& \Theta_{1}=\omega_{1}, \quad \Theta_{2}=\left(\begin{array}{llc}
2-\eta_{21}, & 4+\eta_{11}-\eta_{22}, & -3 \eta_{13}-\eta_{23} \\
0, & 2+\eta_{21}, & -3 \eta_{23} \\
3 \eta_{31}, & \eta_{31}+3 \eta_{32}, & 8
\end{array}\right), \\
& \left(\Theta_{3}\right)_{11}=4-6 \eta_{21}+6 \eta_{13} \eta_{31}+\eta_{23} \eta_{31}+\eta_{21}\left(\eta_{22}-\eta_{11}\right)-\eta_{21}^{\prime}, \\
& \left(\Theta_{3}\right)_{22}=4+6 \eta_{21}+6 \eta_{23} \eta_{32}+\eta_{23} \eta_{31}+\eta_{21}\left(\eta_{11}-\eta_{22}\right)+\eta_{21}^{\prime} \text {, } \\
& \left(\Theta_{3}\right)_{33}=-32-6 \eta_{13} \eta_{31}-2 \eta_{31} \eta_{23}-6 \eta_{23} \eta_{32} \text {, } \\
& \left(\Theta_{3}\right)_{21}=6 \eta_{23} \eta_{31}-2 \eta_{21}^{2} \text {, } \\
& \left(\Theta_{3}\right)_{12}=\left(2+\eta_{11}-\eta_{22}\right)\left(4+\eta_{11}-\eta_{22}\right)+4+2 \eta_{12} \eta_{21}+\eta_{13} \eta_{31}+ \\
& +6 \eta_{13} \eta_{32}+\eta_{32} \eta_{23}+\left(\eta_{11}-\eta_{22}\right)^{\prime} \text {, } \\
& \left(\Theta_{3}\right)_{13}=\eta_{13}\left(\eta_{21}+9-3 \eta_{11}+3 \eta_{33}\right)-3 \eta_{23}\left(2+\eta_{11}+\eta_{12}\right)- \\
& -3 \eta_{13}^{\prime}-\eta_{23}^{\prime} \\
& \left(\Theta_{3}\right)_{23}=\eta_{23}\left(3 \eta_{33}-3 \eta_{22}+9-2 \eta_{21}\right)-3 \eta_{21} \eta_{13}-3 \eta_{23}^{\prime}, \\
& \left(\Theta_{3}\right)_{31}=\eta_{31}\left(3 \eta_{33}-3 \eta_{11}-9-2 \eta_{21}\right)-3 \eta_{21} \eta_{32}+3 \eta_{31}^{\prime} \text {, } \\
& \left(\Theta_{3}\right)_{32}=3 \eta_{31}\left(2-\eta_{22}-\eta_{12}\right)+\eta_{32}\left(3 \eta_{33}-3 \eta_{22}+\eta_{21}-9\right)+ \\
& +3 \eta_{32}^{\prime}+\eta_{31}^{\prime} \text {, } \\
& \vartheta_{1}=0, \quad \vartheta_{2}=\left(\begin{array}{l}
-\eta_{10}-\eta_{20} \\
-\eta_{20} \\
2 \eta_{30}
\end{array}\right), \\
& \vartheta_{3}=\left(\begin{array}{c}
\eta_{10}\left(\eta_{21}-\eta_{11}-3\right)+\eta_{20}\left(\eta_{22}-2 \eta_{11}-\eta_{12}-6\right)+ \\
+\eta_{30}\left(5 \eta_{13}+\eta_{23}\right)-\eta_{10}^{\prime}-\eta_{20}^{\prime} \\
-\eta_{10} \eta_{21}-\eta_{20}\left(3+2 \eta_{21}+\eta_{22}\right)+5 \eta_{30} \eta_{23}-\eta_{20}^{\prime} \\
-4 \eta_{10} \eta_{31}-2 \eta_{20}\left(\eta_{31}+2 \eta_{32}\right)+2 \eta_{30}\left(\eta_{33}-6\right)+2 \eta_{30}^{\prime}
\end{array}\right)
\end{aligned}
$$


Adding equations (2) for the coefficients with the indices $i i, i=1,2,3$, we get $\alpha_{2}=-2$; further, we have $3 \eta_{23} \eta_{31}-\eta_{21}^{2}=0$. The other equations from (2) are

$$
\begin{aligned}
& 8+3 \eta_{13} \eta_{31}+\eta_{31} \eta_{23}+3 \eta_{23} \eta_{32}=\alpha_{1}, \\
& 3 \eta_{13} \eta_{31}-8 \eta_{21}-3 \eta_{23} \eta_{32}+\eta_{21}\left(\eta_{22}-\eta_{11}\right)=\eta_{21}^{\prime}, \\
& \left(4+\eta_{11}-\eta_{22}\right)^{2}-4+\eta_{12} \eta_{21}-2 \eta_{13} \eta_{31}-\eta_{31} \eta_{23}+6 \eta_{13} \eta_{32}- \\
& -2 \eta_{32} \eta_{23}=\left(\eta_{22}-\eta_{11}\right)^{\prime} \\
& \eta_{13}\left(3-3 \eta_{11}+\eta_{21}+3 \eta_{33}\right)-\eta_{23}\left(8+3 \eta_{11}+3 \eta_{12}\right)=3 \eta_{13}^{\prime}+\eta_{23}^{\prime}, \\
& \eta_{23}\left(3 \eta_{33}-3 \eta_{22}+3-2 \eta_{21}\right)-3 \eta_{21} \eta_{13}=3 \eta_{23}^{\prime}, \\
& 3 \eta_{21} \eta_{32}+\eta_{31}\left(3-3 \eta_{33}+3 \eta_{11}+2 \eta_{21}\right)=3 \eta_{31}^{\prime}, \\
& \eta_{31}\left(3 \eta_{22}-3 \eta_{12}-8\right)+\eta_{32}\left(3-3 \eta_{33}+3 \eta_{22}+\eta_{21}\right)=\eta_{31}^{\prime}+3 \eta_{32}^{\prime}, \\
& \eta_{10}^{\prime}=\eta_{10}\left(2 \eta_{21}-\eta_{11}-5\right)+\eta_{20}\left(2 \eta_{21}+2 \eta_{22}-2 \eta_{11}-\eta_{12}-3\right)+ \\
& \quad+\eta_{30}\left(5 \eta_{13}-4 \eta_{23}\right) \\
& \eta_{20}^{\prime}=-\eta_{10} \eta_{21}-\eta_{20}\left(5+2 \eta_{21}+\eta_{22}\right)+5 \eta_{30} \eta_{23} \\
& \eta_{30}^{\prime}=2 \eta_{10} \eta_{31}+\eta_{20}\left(\eta_{31}+2 \eta_{32}\right)+\eta_{30}\left(4-\eta_{33}\right)
\end{aligned}
$$

Now equations (10) must be solved. First we shall try to simplify them by specializing the lift using the group $H_{1}$. To do it, we have to consider various cases.

a) $\eta_{21} \neq 0$. Then also $\eta_{31} \neq 0$ and from (8) we see that the lift of the motion can be changed in such a way that $\eta_{31}=\eta_{21}, \eta_{32}=0$. Then also $3 \eta_{23}=\eta_{21}$. Denote $\eta_{23}=s$. Equations (10) will simplify to $\left(\eta_{21}=\eta_{31}=3 s, \eta_{23}=s, \eta_{32}=0\right)$

$$
\begin{aligned}
& 8+9 \eta_{13} s+3 s^{2}=\alpha_{1}, \quad s\left(3 \eta_{13}-8+\eta_{22}-\eta_{11}\right)=s^{\prime}, \\
& \left(4+\eta_{11}-\eta_{22}\right)^{2}-4+6 \eta_{12} s-6 \eta_{13} s-3 s^{2}+\left(\eta_{11}-\eta_{22}\right)^{\prime}=0, \\
& 3 \eta_{13}\left(1-\eta_{11}+\eta_{33}+s\right)-s\left(8+3 \eta_{11}+3 \eta_{12}\right)=3 \eta_{13}^{\prime}+s^{\prime}, \\
& s\left(1-3 \eta_{13}-2 s+\eta_{33}-\eta_{22}\right)=s^{\prime}, s\left(1+2 s-\eta_{33}+\eta_{11}\right)=s^{\prime} \\
& s\left(3 \eta_{22}+3 \eta_{12}-8\right)=s^{\prime}, \\
& \eta_{10}^{\prime}=\eta_{10}\left(6 s-\eta_{11}-5\right)+\eta_{20}\left(6 s+2 \eta_{22}-2 \eta_{11}-\eta_{12}-3\right)+ \\
& \quad+\eta_{30}\left(5 \eta_{13}-4 s\right) \\
& \eta_{20}^{\prime}=-3 \eta_{10} s-\eta_{20}\left(5+6 s+\eta_{22}\right)+5 \eta_{30} s \\
& \eta_{30}^{\prime}=6 \eta_{10} s+3 \eta_{20} s+\eta_{30}\left(4-\eta_{33}\right) .
\end{aligned}
$$

The sum of the second, fifth and sixth equation from (11) gives $s^{\prime}=-2 s$. Substitution in the second, fifth and seventh equation in (11) gives $\eta_{12}=2-\eta_{22}$, $\eta_{11}=-\frac{1}{2}\left(3+2 s+\eta_{22}\right), \quad \eta_{13}=\frac{1}{6}\left(9-2 s-3 \eta_{22}\right)$. Substitution in the fourth and third equation in (11) gives $3 \eta_{22}^{\prime}=3-10 \eta_{22}+3 \eta_{22}^{2}, \eta_{22}^{\prime}=4 \eta_{22}-12$. Hence we have $\eta_{22}^{2}-6 \eta_{22}+9=0$, so $\eta_{22}=$ const., $\eta_{22}^{\prime}=0$ and the only possibility for $\eta_{22}$ is $\eta_{22}=3$. The result is 


$$
\eta_{11}=-s-3, \quad \eta_{12}=-1, \quad \eta_{13}=-\frac{s}{3}, \quad \eta_{33}=s, \quad \alpha_{1}=8
$$

The equations for $\eta_{0}$ are

$$
\begin{aligned}
& \eta_{10}^{\prime}=(7 s-2) \eta_{10}+(8 s+10) \eta_{20}-\frac{17}{3} s \eta_{30}, \\
& \eta_{20}^{\prime}=-3 s \eta_{10}-(6 s+8) \eta_{20}+5 s \eta_{30}, \\
& \eta_{30}^{\prime}=6 s \eta_{10}+3 s \eta_{20}+(4-s) \eta_{30} .
\end{aligned}
$$

The solution for $\eta_{0}$ can be written in the form $\eta_{0}=\sum_{i=1}^{3} C_{i} \mu_{i}$, where $C_{i}$ are constants of integration and

$$
\mu_{1}=\left(\begin{array}{l}
-s^{-1} \\
3 s^{-1} \\
3 s^{-1}+6 s^{-2}
\end{array}\right), \quad \mu_{2}=\left(\begin{array}{c}
-s^{5}-10 s^{4} \\
3 s^{5}+6 s^{4} \\
3 s^{5}
\end{array}\right), \quad \mu_{3}=\left(\begin{array}{c}
-s^{2}-4 s \\
3 s^{2} \\
3 s^{2}
\end{array}\right) .
$$

Let now $\eta_{21}=0$. Then $\eta_{23} \eta_{31}=0$. The first two equations from (10) give $8+$ $+6 \eta_{13} \eta_{31}=8+6 \eta_{23} \eta_{32}=\alpha_{1}$. If $\eta_{31} \neq 0$, then $\eta_{23}=0$ and $\alpha_{1}=8$. If $\eta_{31}=0$, then also $\alpha_{1}=8$. So in all the following cases we have $\alpha_{2}=-2, \alpha_{1}=8$ and $\eta_{23} \eta_{31}=$ $=\eta_{13} \eta_{31}=\eta_{23} \eta_{32}=0$.

b) $\eta_{21}=0, \eta_{31} \neq 0$. Then $\eta_{23}=\eta_{13}=0$; we can specialize the lift of the motion to get $\eta_{32}=0, \eta_{31}=1$. Equations (10) will now be

$$
\left(4+\eta_{11}-\eta_{22}\right)^{2}+\left(\eta_{11}-\eta_{22}\right)^{\prime}=4, \quad 3 \eta_{12}+3 \eta_{22}=8, \quad \eta_{33}-\eta_{11}=1 .
$$

Let $u$ be any solution of the differential equation $u^{\prime}+3 u^{2}-4 u=0$. Then either $u=\frac{4}{3}\left(1-s^{2}\right)^{-1}$, where $s^{\prime}=-2 s$, or $u=0$. Further, $\eta_{11}-\eta_{22}=-6+3 u$ and so $\eta_{11}=-\frac{7}{3}+u, \eta_{22}=\frac{11}{3}-2 u, \eta_{33}=-\frac{4}{3}+u, \eta_{12}=-1+2 u$, the others vanish. $\eta_{0}$ can be written similarly as in a), where

$$
\mu_{1}=u^{1 / 3}\left(\begin{array}{c}
4 s^{2} \\
0 \\
-s^{2}
\end{array}\right), \quad \mu_{2}=u^{1 / 3}\left(\begin{array}{c}
2 s^{3}\left(1+5 s^{2}\right) \\
6 s^{3}\left(1-s^{2}\right) \\
-s^{3}\left(1+s^{2}\right)
\end{array}\right), \quad \mu_{3}=u^{1 / 3}\left(\begin{array}{c}
0 \\
0 \\
-s^{-2}
\end{array}\right)
$$

for $u \neq 0$ and

$$
\mu_{1}=\exp \left(-\frac{8}{3} t\right)\left(\begin{array}{r}
4 \\
0 \\
-1
\end{array}\right), \quad \mu_{2}=\exp \left(-\frac{26}{3} t\right)\left(\begin{array}{r}
-10 \\
6 \\
1
\end{array}\right), \quad \mu_{3}=\exp \left(\frac{16}{3} t\right)\left(\begin{array}{l}
0 \\
0 \\
1
\end{array}\right)
$$

for $u=0$.

c) $\eta_{21}=\eta_{31}=0, \eta_{23} \neq 0$. Then $\eta_{32}=0$ and we specialize to $\eta_{23}=1, \eta_{13}=0$. Equations (10) will be

$$
\left(4+\eta_{11}-\eta_{22}\right)^{2}+\left(\eta_{11}-\eta_{22}\right)^{\prime}=4, \quad 3 \eta_{11}+3 \eta_{12}=-8, \quad \eta_{22}-\eta_{33}=1 .
$$


Let $u$ be as in the case b). Then

$$
\begin{aligned}
& \eta_{11}=-\frac{11}{3}+2 u, \quad \eta_{22}=\frac{7}{3}-u, \quad \eta_{33}=\frac{4}{3}-u, \quad \eta_{12}=1-2 u, \\
& \eta_{10}=\frac{1}{3}\left(4 C_{2} s^{5}-4 C_{2} s^{3}-C_{3} s^{-2}+C_{1} s^{2}\right)\left(1-s^{2}\right)^{-2 / 3}, \\
& \eta_{20}=\frac{1}{2}\left(C_{3} s^{-2}+2 C_{2} s^{3}\right)\left(1-s^{2}\right)^{1 / 3}, \quad \eta_{30}=C_{3} s^{-2}\left(1-s^{2}\right)^{1 / 3}
\end{aligned}
$$

for $u \neq 0$,

$$
\begin{aligned}
& \eta_{10}=-\frac{4}{3} C_{2} \exp \left(-\frac{22}{3} t\right)+C_{1} \exp \left(-\frac{4}{3} t\right), \\
& \eta_{20}=\frac{1}{2} C_{3} \exp \left(\frac{8}{3} t\right)+C_{2} \exp \left(-\frac{22}{3} t\right), \\
& \eta_{30}=C_{3} \exp \left(\frac{8}{3} t\right)
\end{aligned}
$$

for $u=0$.

d) $\eta_{21}=\eta_{31}=\eta_{23}=0$. Let $\eta_{13} \neq 0, \eta_{32} \neq 0$. Then we can specialize to $\eta_{32}=$ $=\varepsilon \eta_{13}, \eta_{13}>0, \varepsilon= \pm 1$. No further specialization is possible, so we put $\eta_{12}=0$, the lift is fixed up to a constant matrix. Equations (10) will be

$$
\begin{gathered}
\left(4+\eta_{11}-\eta_{22}\right)^{2}+\left(\eta_{11}-\eta_{22}\right)^{\prime}+6 \varepsilon \eta_{13}^{2}=4, \\
\eta_{13}\left(1-\eta_{11}+\eta_{33}\right)=\eta_{13}\left(-1+\eta_{33}-\eta_{22}\right)=\eta_{13}^{\prime} .
\end{gathered}
$$

First we obtain $\eta_{33}=0, \eta_{22}=-\eta_{11}, \eta_{13}^{\prime} \eta_{13}^{-1}=1-\eta_{11}$. Denote $\eta_{13}=v^{-1 / 2}$. Then $v$ must be a solution of $v^{\prime \prime}+12 v^{\prime}+32 v=-6 \varepsilon$. Further, we get

$$
\begin{aligned}
& \eta_{11}=1+\frac{1}{2} v^{\prime} v^{-1}, \quad \eta_{22}=-1-\frac{1}{2} v^{\prime} v^{-1}, \quad \eta_{32}=\varepsilon v^{-1 / 2}, \\
& \eta_{10}^{\prime}=-\eta_{10}\left(6+\frac{1}{2} v^{\prime} v^{-1}\right)-\eta_{20}\left(7+2 v^{\prime} v^{-1}\right)+5 \eta_{30} v^{-1 / 2}, \\
& \eta_{20}^{\prime}=\eta_{20}\left(-4+\frac{1}{2} v^{\prime} v^{-1}\right), \quad \eta_{30}=2 \eta_{20} \varepsilon v^{-1 / 2}+4 \eta_{30} .
\end{aligned}
$$

Denote $H(t)=\int_{t_{0}}^{t} \mathrm{e}^{2 z} v(z) \mathrm{d} z$ for a suitable $t_{0}$. Then

$$
\begin{aligned}
& \eta_{10}=\left(-3 C_{2} H-2 C_{2} \mathrm{e}^{2 t} v-\frac{5}{8} \varepsilon C_{2} \mathrm{e}^{2 t}+\frac{1}{2} C_{3} \mathrm{e}^{10 t}+C_{1}\right) \mathrm{e}^{-6 t} v^{-1 / 2}, \\
& \eta_{20}=C_{2} \mathrm{e}^{-4 t} v^{1 / 2}, \quad \eta_{30}=-\varepsilon / 4 C_{2} \mathrm{e}^{-4 t}+C_{3} \mathrm{e}^{4 t} .
\end{aligned}
$$

e) $\eta_{21}=\eta_{31}=\eta_{23}=\eta_{32}=0, \eta_{13} \neq 0$. Then we get $\eta_{12}=0, \eta_{13}=1$ by specialization again up to a constant matrix only. Denote $u$ as in the case b). Then $\eta_{11}=-\frac{5}{3}+u, \eta_{22}=\frac{13}{3}-2 u, \eta_{33}=-\frac{8}{3}+u$,

$$
\begin{gathered}
\eta_{10}=\frac{1}{2}\left(3 C_{2} s^{16 / 3}-C_{2} s^{10 / 3}+\frac{1}{2} C_{3} s^{-8 / 3}+C_{1} s^{7 / 3}\right)\left(1-s^{2}\right)^{-1 / 3}, \\
\eta_{20}=C_{2} s^{10 / 3}\left(1-s^{2}\right)^{2 / 3}, \quad \eta_{30}=C_{3} s^{-8 / 3}\left(1-s^{2}\right)^{-1 / 3}
\end{gathered}
$$

for $u \neq 0$ and

$$
\begin{gathered}
\eta_{10}=C_{1} \exp \left(-\frac{10}{3} t\right)-\frac{4}{3} C_{2} \exp \left(-\frac{28}{3} t\right)+\frac{1}{2} C_{3} \exp \left(\frac{20}{3} t\right), \quad \eta_{20}=C_{2} \exp \left(-\frac{28}{3} t\right), \\
\eta_{30}=C_{3} \exp \left(\frac{20}{3} t\right)
\end{gathered}
$$

for $u=0$. 
f) $\eta_{i j}=0$ for $i \neq j, i, j=1,2,3$. Then we can also suppose $\eta_{11}=0, \eta_{33}=-\eta_{22}$ : Let $u$ be as in the case b). Then $\eta_{22}=6-3 u$,

$$
\begin{aligned}
& \eta_{10}=\mathrm{e}^{-t}\left[C_{1} s^{2}+\frac{1}{2} C_{2}\left(3 s^{5}-s^{3}\right)\right], \\
& \eta_{20}=C_{2} \mathrm{e}^{-t} s^{3}\left(1-s^{2}\right), \quad \eta_{30}=C_{3} s^{-3}\left(1-s^{2}\right)^{-1}
\end{aligned}
$$

for $u \neq 0$ and

$$
\eta_{10}=C_{1} \mathrm{e}^{-5 t}-\frac{3}{2} C_{2} \mathrm{e}^{-11 t}, \quad \eta_{20}=C_{2} \mathrm{e}^{-11 t}, \quad \eta_{30}=C_{3} \mathrm{e}^{10 t}
$$

for $u=0$.

This covers all subcases of i). In all of them we have $\alpha_{2}=-2, \alpha_{1}=8$. This means that the differential equation for the trajectories of points is $X^{\prime \prime \prime}=-2 X^{\prime \prime}+8 X^{\prime}$. The solution is $X=A_{0}+e_{1} \mathrm{e}^{-4 t}+e_{2} \mathrm{e}^{2 t}$, where $\left\{A_{0}, e_{1}, e_{2}\right\}$ is a suitable frame in the affine plane. All such curves are affinely equivalent to the curve $y=1 / x^{2}$.

Differential equations for the Frenet frames $\overline{\mathscr{R}}=\left\{\bar{A}, \bar{e}_{1}, \bar{e}_{2}, \bar{e}_{3}\right\}$ in the moving space and $\mathscr{R}=\left\{A, e_{1}, e_{2}, e_{3}\right\}$ in the fixed space of the motion are

$$
\overline{\mathscr{R}}^{\prime}=\overline{\mathscr{R}}(\eta-\omega), \quad \mathscr{R}^{\prime}=\mathscr{R}(\eta+\omega), \text { respectively . }
$$

The Frenet frames $\overline{\mathscr{R}}$ and $\mathscr{R}$ are the canonical (or Frenet) frames of the moving and fixed axoids of the motion respectively. This means that $A(t)$ is the poloid, $A(t)+$ $+\lambda e_{1}(t), A(t)+\lambda e_{3}(t)$ are two ruled surfaces, $A(t)+\lambda e_{1}(t)+\mu e_{2}(t)$ is a system of planes and similarly for the moving space. These objects describe the motion similarly as axoids do in the case of a Euclidean space motion. To find explicit expressions for axoids we have to solve (12) in all subcases a) $-\mathrm{f}$ ). For the sake of simplicity we shall write these solutions in the form of matrices $g_{1}$ and $g_{2}$, where

$$
\mathscr{R}=\mathscr{R}_{0} \cdot g_{1}, \quad \overline{\mathscr{R}}=\overline{\mathscr{R}}_{0} \cdot g_{2} .
$$

Further, we write

where

$$
g_{1}=\left(\begin{array}{ll}
1, & 0 \\
T_{1}, & \gamma_{1}
\end{array}\right), \quad g_{2}=\left(\begin{array}{ll}
1, & 0 \\
T_{2}, & \gamma_{2}
\end{array}\right)
$$

$T_{1}=A_{0}+\sum_{i=1}^{3} C_{i} A_{i}, T_{2}=\bar{A}_{0}+\sum_{i=1}^{3} C_{i} \bar{A}_{i}$, and $C_{i}$ are constants of integration specified above. Solutions of (12) are the following: (in b), c), e), f) for $u \neq 0$ only):

a)

$$
\begin{aligned}
& \gamma_{1}=\left(\begin{array}{lll}
s+\frac{1}{2} s^{2}, & 0, & \frac{1}{6} s^{2} \\
\frac{3}{4} s^{-1}, & s^{-2}, \frac{1}{4} s^{-1} \\
3 s, & 0, & s
\end{array}\right), \quad \gamma_{2}=\left(\begin{array}{lll}
3 s^{2}, & s^{2}, & 0 \\
\frac{1}{4}, & \frac{1}{3} s^{-1}+\frac{1}{4}, & -\frac{1}{6} \\
0, & -s^{-1}, & s^{-1}
\end{array}\right), \\
& A_{1}=\left(\begin{array}{l}
0 \\
3 s^{-3} \\
12 s^{-1}
\end{array}\right), \quad A_{2}=\left(\begin{array}{c}
12 s^{5}+6 s^{6} \\
9 s^{3}-18 s^{2} \\
36 s^{5}
\end{array}\right), \quad A_{3}=\left(\begin{array}{l}
2 s^{2}+s^{3} \\
0 \\
6 s^{3}
\end{array}\right), \\
& \bar{A}_{1}=\left(\begin{array}{l}
24 s^{6} \\
0 \\
4 s^{-3}
\end{array}\right), \quad \bar{A}_{2}=\left(\begin{array}{l}
4 s^{3} \\
12 s^{3}
\end{array}\right), \quad \bar{A}_{3}=\left(\begin{array}{l}
0 \\
0
\end{array}\right) .
\end{aligned}
$$


b)

$$
\begin{gathered}
\gamma_{1}=\frac{1}{12}\left(1-s^{2}\right)^{1 / 3}\left(\begin{array}{ccc}
12, & -16\left(1-s^{2}\right)^{-1}, & 0 \\
0, & 12 s^{-1}\left(1-s^{2}\right)^{-1}, & 0 \\
-6 s, & 4 s\left(1-s^{2}\right)^{-1}, & 12 s
\end{array}\right), \\
\gamma_{2}=\frac{1}{12}\left(1-s^{2}\right)^{1 / 3}\left(\begin{array}{ccc}
12 s, & -4 s\left(1+s^{2}\right)\left(1-s^{2}\right)^{-1}, & 0 \\
0, & 12\left(1-s^{2}\right)^{-1}, & 0 \\
3 s^{-1}, & s^{-1}\left(1-3 s^{2}\right)\left(1-s^{2}\right)^{-1}, & 12 s^{-1}
\end{array}\right), \\
A_{1}=\left(\begin{array}{c}
6 s^{2} \\
0 \\
-3 s^{3}
\end{array}\right), \quad A_{2}=\left(\begin{array}{c}
-6 s^{3}+6 s^{5} \\
9 s^{2} \\
-3 s^{6}
\end{array}\right), \quad A_{3}=\left(\begin{array}{l}
0 \\
0 \\
3 s^{-1}
\end{array}\right), \\
\bar{A}_{1}=\left(\begin{array}{l}
4 s^{3} \\
0 \\
0
\end{array}\right), \quad \bar{A}_{2}=\left(\begin{array}{l}
4 s^{6} \\
6 s^{3} \\
0
\end{array}\right), \quad \bar{A}_{3}=\left(\begin{array}{l}
0 \\
0 \\
s^{-3}
\end{array}\right) .
\end{gathered}
$$

c) $\quad \gamma_{1}=\frac{1}{12}\left(1-s^{2}\right)^{-1 / 3}\left(\begin{array}{ccl}12\left(1-s^{2}\right), & 4\left(1+s^{2}\right) & 2\left(1-s^{2}\right) \\ 0, & 12 s^{-1}, & 3 s^{-1} \\ 0, & 0, & 12 s\end{array}\right)$,

$$
\begin{gathered}
\gamma_{2}=\frac{1}{12}\left(1-s^{2}\right)^{-1 / 3}\left(\begin{array}{ccc}
12 s\left(1-s^{2}\right), & 16 s, & -4 s \\
0, & 12, & -6 \\
0, & 0, & 12 s^{-1}
\end{array}\right), \\
A_{1}=\left(\begin{array}{l}
3 s^{2} \\
0 \\
0
\end{array}\right), \quad A_{2}=\left(\begin{array}{l}
2 s^{5}-2 s^{3} \\
3 s^{2} \\
0
\end{array}\right), \quad A_{3}=\left(\begin{array}{l}
0 \\
\frac{3}{2} s^{-3} \\
6 s^{-1}
\end{array}\right), \\
\bar{A}_{1}=\left(\begin{array}{l}
2 s^{3} \\
0 \\
0
\end{array}\right), \quad \bar{A}_{2}=\left(\begin{array}{l}
\frac{4}{3} s^{6} \\
2 s^{3} \\
0
\end{array}\right), \quad \bar{A}_{3}=\left(\begin{array}{l}
0 \\
0 \\
2 s^{-3}
\end{array}\right) .
\end{gathered}
$$

d)

$$
\begin{aligned}
& \gamma_{1}=\frac{1}{8} v^{-1 / 2}\left(\begin{array}{lcl}
8 \mathrm{e}^{2 t} v, & 8 H+\varepsilon \mathrm{e}^{2 t}, & 2 \mathrm{e}^{2 t} v^{1 / 2} \\
0, & 8, & 0 \\
0, & -4 \varepsilon \mathrm{e}^{-2 t}, & 8 \mathrm{e}^{-2 t} v^{1 / 2}
\end{array}\right), \\
& \gamma_{2}=\frac{1}{8} v^{-1 / 2}\left(\begin{array}{ccc}
8 v, & -8 H-2 \varepsilon, & -4 v^{1 / 2} \\
0, & 8 \mathrm{e}^{-2 t}, & 0 \\
0, & 2 \varepsilon \mathrm{e}^{2 t}, & 8 \mathrm{e}^{2 t} v^{1 / 2}
\end{array}\right), \\
& A_{1}=\left(\begin{array}{l}
3 \mathrm{e}^{-4 t} \\
0 \\
0
\end{array}\right), \quad A_{2}=\left(\begin{array}{c}
16 K+16 L-\frac{9}{4} \varepsilon \mathrm{e}^{-2 t} \\
2 \mathrm{e}^{-4 t} \\
\mathrm{e}^{-6 t}
\end{array}\right), \quad A_{3}=\left(\begin{array}{l}
\frac{3}{2} \mathrm{e}^{6 t} \\
0 \\
6 \mathrm{e}^{2 t}
\end{array}\right) \text {, } \\
& \bar{A}_{1}=\left(\begin{array}{l}
2 \mathrm{e}^{-6 t} \\
0 \\
0
\end{array}\right), \quad \bar{A}_{2}=\left(\begin{array}{l}
32 M+16 N-\frac{3}{2} \varepsilon \mathrm{e}^{-4 t} \\
\frac{4}{3} \mathrm{e}^{-6 t} \\
0
\end{array}\right), \quad \bar{A}_{3}=\left(\begin{array}{l}
0 \\
0 \\
2 \mathrm{e}^{6 t}
\end{array}\right),
\end{aligned}
$$


where $M=\int_{t_{0}}^{t} H(z) \mathrm{e}^{-6 z} \mathrm{~d} z, \quad N=\int_{t_{0}}^{t} v(z) \mathrm{e}^{-4 z} \mathrm{~d} z, \quad K=\int_{t_{0}}^{t} H(z) \mathrm{e}^{-4 z} \mathrm{~d} z, \quad L=$ $=\int_{t_{0}}^{t} v(z) \mathrm{e}^{-2 z} \mathrm{~d} z$.

e) $\quad \gamma_{1}=\frac{1}{6} s^{-1 / 3}\left(1-s^{2}\right)^{1 / 3}\left(\begin{array}{ll}6,\left(s^{2}-3\right)\left(1-s^{2}\right)^{-1}, & \frac{3}{2} \\ 0,6 s^{-1}\left(1-s^{2}\right)^{-1}, & 0 \\ 0, & 0,\end{array}\right)$,

$$
\begin{gathered}
\gamma_{2}=\frac{1}{6} s^{-1 / 3}\left(1-s^{2}\right)^{1 / 3}\left(\begin{array}{lll}
6 s, & \left(3 s-s^{3}\right)\left(1-s^{2}\right)^{-1}, & -3 s \\
0, & 6\left(1-s^{2}\right)^{-1}, & 0 \\
0, & 0, & 6
\end{array}\right), \\
A_{3}=\left(\begin{array}{l}
\frac{3}{2} s^{-3} \\
0 \\
6 s^{-1}
\end{array}\right), \text { the others as in c). }
\end{gathered}
$$

f) $\gamma_{1}=\frac{1}{6} \mathrm{e}^{t}\left(\begin{array}{ll}6,\left(s^{2}-3\right)\left(1-s^{2}\right)^{-1}, & 0 \\ 0,6 s^{-1}\left(1-s^{2}\right)^{-1}, & 0 \\ 0,0, & 6 s^{2}\left(1-s^{2}\right) \mathrm{e}^{-t}\end{array}\right)$.

$$
\gamma_{2}=\frac{1}{6} \mathrm{e}^{-t}\left(\begin{array}{ll}
6,\left(s^{2}-3\right)\left(1-s^{2}\right)^{-1}, & 0 \\
0,6 s^{-1}\left(1-s^{2}\right)^{-1}, & 0 \\
0,0, & 6\left(1-s^{2}\right) \mathrm{e}^{t}
\end{array}\right)
$$

$$
A_{3}=\left(\begin{array}{l}
0 \\
0 \\
6 s^{-1}
\end{array}\right) \text {, the others as in c). }
$$

Now for $u=0$ :

b) $\gamma_{1}=s^{2 / 3}\left(\begin{array}{ccc}1, & 0, & 0 \\ 0, & s^{-3}, & 0 \\ -\frac{1}{2} s, & 0, & s\end{array}\right), \quad \gamma_{2}=s^{2 / 3}\left(\begin{array}{lll}s, & \frac{1}{3} s, & 0 \\ 0, & s^{-2}, & 0 \\ \frac{1}{4} s^{-1}, & \frac{1}{4} s^{-1}, & s^{-1}\end{array}\right)$,

$$
A_{2}=\left(\begin{array}{r}
6 s^{5} \\
9 s^{2} \\
-3 s^{6}
\end{array}\right), \quad \bar{A}_{2}=\left(\begin{array}{c}
4 s^{6} \\
-6 s^{3} \\
0
\end{array}\right)
$$

the others as in b) for $u \neq 0$.

c) $\gamma_{1}=s^{1 / 3}\left(\begin{array}{ccc}s, & -\frac{1}{3} s, & \frac{1}{6} s \\ 0, & s^{-2}, & \frac{1}{4} s^{-2} \\ 0, & 0, & 1\end{array}\right), \quad \gamma_{2}=s^{1 / 3}\left(\begin{array}{ccc}s^{2}, & 0, & 0 \\ 0, & s^{-1}, & -\frac{1}{2} s^{-1} \\ 0, & 0, & s^{-2}\end{array}\right)$,

$$
A_{2}=\left(\begin{array}{c}
6 s^{5} \\
-9 s^{2} \\
0
\end{array}\right), \quad \bar{A}_{2}=\left(\begin{array}{c}
4 s^{6} \\
-6 s^{3} \\
0
\end{array}\right)
$$

the others as in c) for $u \neq 0$. 
e) $\gamma_{1}=s^{1 / 3}\left(\begin{array}{lll}1, & -\frac{1}{6}, & \frac{1}{4} \\ 0, & s^{-3}, & 0 \\ 0, & 0, & s^{2}\end{array}\right)$ $\gamma_{2}=s^{1 / 3}\left(\begin{array}{llc}s, & \frac{1}{6} s, & -\frac{1}{2} s \\ 0, & s^{-2}, & 0 \\ 0, & 0, & 1\end{array}\right), \quad A$ is as in e) for $u \neq 0$.

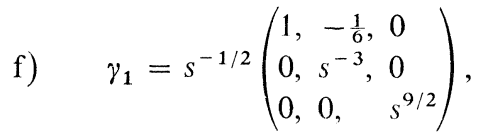

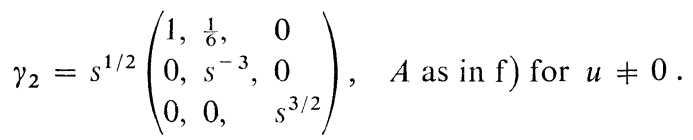

Now $g=g_{1} g_{2}^{-1}$ is the matrix of the motion. Write

$$
g=\left(\begin{array}{ll}
1, & 0 \\
T, & \gamma
\end{array}\right)
$$

Then $\gamma=\gamma_{1} \gamma_{2}^{-1}, T=T_{1}-\gamma T_{2}$. Choosing suitably $A_{0}$ and $\bar{A}_{0}$ we can write $T=$ $=\sum_{i=1}^{3} C_{i} t_{i}$. The result is:

$$
\text { a) } \gamma=\left(\begin{array}{ccc}
\frac{1}{3} s^{-1}+\frac{1}{4}, & -s^{2}, & 0 \\
0, & 3 s^{-1}, & \frac{3}{4} \\
s^{-1}, & 0, & s^{2}
\end{array}\right), \quad t_{1}=\left(\begin{array}{l}
0 \\
0 \\
s^{-1}
\end{array}\right), \quad t_{2}=\left(\begin{array}{l}
0 \\
s^{2} \\
0
\end{array}\right), \quad t_{3}=\left(\begin{array}{l}
s^{2} \\
0 \\
3 s^{2}
\end{array}\right)
$$

b) c) e) f) $\gamma=\left(\begin{array}{ccc}s^{-1}, & -1+\frac{1}{3} s^{2}, & \frac{3}{4} v_{3} \\ 0, & s^{-1}, & \frac{3}{4} v_{2} \\ -\frac{3}{4} v_{1}, & 0, & s^{2}\end{array}\right)$ for $u \neq 0$

$$
\begin{gathered}
\gamma=\left(\begin{array}{ccc}
s^{-1}, & -\frac{1}{3} s^{2}, & \frac{3}{4} v_{3} \\
0, & s^{-1}, & \frac{3}{4} v_{2} \\
-\frac{3}{4} v_{1}, & 0, & s^{2}
\end{array}\right) \text { for } u=0 . \\
t_{1}=\left(\begin{array}{l}
s^{2} \\
0 \\
0
\end{array}\right), \quad t_{2}=\left(\begin{array}{l}
0 \\
s^{2} \\
0
\end{array}\right), \quad t_{3}=\left(\begin{array}{l}
0 \\
0 \\
s^{-1}
\end{array}\right), \quad \text { where } v_{1}, v_{2}, v_{3} \text { are either } 1 \text { or } 0
\end{gathered}
$$

and at most one of them is equal to 1 .

d) $\gamma=\left(\begin{array}{lll}s^{-1}, & D_{1}+D_{2} s^{2}, \frac{3}{4} \\ 0, & s^{-1}, & 0 \\ 0, & \frac{3}{4} \varepsilon, & s^{2}\end{array}\right)$, where $D_{1}, D_{2}$ are any constants, $\varepsilon= \pm 1$ and $T$ is the same as in b). 
Theorem 1. Every equiaffine Darboux motion with the instantaneous motion preserving exactly two intersecting lines and a plane containing one of them is equivalent to one of those listed in (14).

Now we have to do the same for the case ii. As the procedure is very similar, we present only the results.

Theorem 2. Every equiaffine Darboux motion with the instantaneous motion preserving a pencil of lines and one more line through its center is equivalent to one from (15):

$$
\gamma=\left(\begin{array}{ccl}
s, & 0, & \frac{3}{4} v_{2} \\
0, & s, & 0 \\
-\frac{3}{4} v_{1}, & 0, & s^{-2}
\end{array}\right), \quad T=\left(\begin{array}{l}
C_{1} s^{-2} \\
C_{2} s^{-2} \\
C_{3} s
\end{array}\right),
$$

where $v_{1}, v_{2}$ are either 1 or 0 and at most one of them is equal to $1, C_{i}, i=1,2,3$ are constants.

Theorem 3. Every equiaffine Darboux motion with the tangent operator having a double characteristic root has trajectories of all points affinely equivalent to the curve $y=x^{-2}$.

\section{References}

[1] Blaschke W.: Zur Kinematik. Abh. math. Sem. Univ. Hamburg, 22 (1958), 171-175.

[2] Karger A.: Darboux motions in $E_{n}$, Czech. Math. Journ., 29 (104) (1979), 303-317.

Author's address: 18000 Praha 8 - Karlín, Sokolovská 83, ČSSR (Matematicko-fyzikální fakulta UK). 